\title{
Redefining Medical Competencies for an Oral Medicine Specialty Training Curriculum Using a Modified Delphi Technique
}

\begin{abstract}
Philip A. Atkin, Roddy McMillan
Abstract: This article describes the development of medical competencies for oral medicine specialty training in the UK and Ireland by a collaborative working group using a modified Delphi technique. The current specialty training curriculum for oral medicine $(\mathrm{OM})$ in the UK was developed by a working group including members of the British Society for Oral Medicine (BSOM) and members of the Specialty Advisory Committee for Additional Dental Specialties (SACADS) and adopted by the UK General Dental Council (GDC) in 2010. When the curriculum was developed, the entry requirements for specialty training in $\mathrm{OM}$ included undergraduate degrees in both dentistry and medicine. At the time of adoption, the requirement for a medical degree was removed. Medical competencies were assumed to have been delivered in medical undergraduate and postgraduate training. Accordingly, there was a need to define the medical competencies for OM specialty training to benefit trainees, trainers, and assessors. In 2018, a group comprising specialty trainers, recent former specialty trainees, and current specialty trainees in OM held face-to-face meetings in addition to email discussions and developed an updated curriculum document to better reflect the medical competencies required in specialty training. A collaborative modified Delphi approach was used to evaluate medical foundation competencies and to include only those that were considered relevant to OM specialty training. A list of relevant and achievable medical competencies was determined that has been approved by SACADS and will be incorporated into a revised OM curriculum from the UK GDC. The newly agreed-upon document for medical competencies in OM specialty training will serve as a reference for trainees, trainers, and assessors and reflects a successful use of a modified Delphi approach.
\end{abstract}

Philip A. Atkin, MSc, FDS (Eng), MFDS, FDS (OM), RCPSG, FHEA, is Consultant and Hon. Senior Lecturer in Oral Medicine, School of Dentistry, Cardiff University, University Hospital of Wales, United Kingdom; and Roddy McMillan, MFDS, FDS (OM), RCPSG, FHEA, is Consultant and Hon. Clinical Teaching Fellow in Oral Medicine, Eastman Dental Institute, University College London, United Kingdom. Direct correspondence to Dr. Philip A. Atkin, School of Dentistry, Cardiff University, University Hospital of Wales, Heath Park, Cardiff CF14 4XY, United Kingdom; +44 292074 2463; atkinpa@cardiff.ac.uk.

Keywords: advanced dental education, oral medicine, curriculum, competencies

Submitted for publication 3/8/19; accepted 6/20/19; first published online 8/12/19 doi: 10.21815/JDE.019.137

$\mathrm{T}$ This article describes the development of and agreement to a set of medical competencies for oral medicine specialty training in the United Kingdom (UK) and Ireland by a collaborative working group using a modified Delphi technique. The aim was to supplement the existing specialty training curriculum published by the UK General Dental Council (GDC) in 2010. ${ }^{1}$ These medical competencies, and the Delphi method of selection, may be useful for similar oral medicine training programs outside of the UK. In the UK, the GDC defines the specialty of oral medicine as "oral health care of patients with chronic recurrent and medically related disorders of the mouth and with their diagnosis and non-surgical management." It is a specialty that looks after those under its care in outpatient settings, and its practitioners will liaise and advise where needed with medical and surgical colleagues for the care of inpatients.
Prior to its publication in 2010, the draft oral medicine $(\mathrm{OM})$ curriculum was developed by a working group with stakeholders representing OM consultants and trainers from the UK and Ireland, OM specialty trainees, educational experts, and a lay representative. ${ }^{1}$ The draft curriculum was submitted to the Specialty Advisory Committee for Additional Dental Specialties (SACADS) for consideration and ratification prior to submission and publication by the GDC. The SACADS consists of four specialty subgroups: oral medicine, oral microbiology, dental and maxillofacial radiology, and oral and maxillofacial pathology. Each of the four SACADS subgroups acts as a mini-SAC for its specialty.

The current (2010) OM curriculum was prepared over approximately two years and comprises 54 pages of detail on training outcomes and assessment. ${ }^{1}$ Its early development was informed by a two-page curriculum document produced by the 
Joint Advisory Committee for the Additional Dental Specialties (JACADS), the predecessor of SACADS, and published in 1999. The JACADS document described the entry criteria into specialty training in $\mathrm{OM}$ and mandated the possession of both dental and medical qualifications and training, allowing full registration with the GDC and UK General Medical Council (GMC). Whilst the 2010 GDC OM curriculum is in most areas extremely detailed, some of the medical competencies are less well defined-the assumption being that they would be gained through formal medical education and training. In the 2010 OM curriculum, entry to specialty training no longer mandates possession of a registerable medical qualification as an essential requirement, and the length of OM specialty training is increased to five years, with an allowance of two years for those trainees with a registerable medical qualification. The competencies in the OM curriculum remained the same as they had been prior to 2010 .

In the years following introduction of the 2010 GDC OM specialist list, specialty registrars in possession of a dental qualification or both medical and dental qualifications continued to be accepted into OM training programs. Postgraduate dental deans, training program directors, and specialty trainees have used GDC curriculum guidance to inform training content, outcomes, and assessments. Specialty trainees complete workplace-based assessments as part of training and are subject to regular review in the Annual Review of Competence Progression (ARCP) process. Towards the end of training and prior to application for entry to the GDC specialist list in $\mathrm{OM}$, trainees must take the Intercollegiate Specialty Fellowship Examination (ISFE) in oral medicine. This exam is coordinated by the dental faculties of the four Royal Colleges of Surgeons in England, Scotland (Glasgow and Edinburgh), and Ireland. The ISFE has a clinical component and uses the GDC OM curriculum to identify areas for assessment. As time has passed, specialty trainees, training program directors, and ISFE examination boards have sought greater clarity on what medical competencies ought to be included in an OM training program and therefore also to be considered for the $\mathrm{ARCP}$ and in the ISFE assessment.

In 2019, the GDC published an updated version of its 2015 "Standards for Specialty Education," which includes the statement, "For clinical procedures, the program provider should be assured that the specialty trainee is safe to treat patients in the relevant skills at the levels required prior to treating patients." It is incumbent on trainers and trainees in oral medicine to make sure that the skills, including medical skills, are appropriate and defined in the training program. The apparent lack of clarity on medical competencies was becoming a problem, and a group of OM clinicians came together with the aim of bringing greater clarity to the issue and adopting robust methods to identify and adopt these competencies. These defined medical competencies will guide trainees, their consultant trainers, training program directors, and postgraduate dental deans who are ultimately responsible for delivering a comprehensive specialty training program in OM. This article describes the process of developing these medical competencies for $\mathrm{OM}$ and its outcomes.

\section{Methods}

In 2018, a group of consultant trainers and specialty trainees in OM organized a working group to develop greater clarity in the medical competencies that ought to be included in OM specialty training programs. This group produced an agreed-upon list of these competencies to aid all interested parties. The working group was a subgroup of the British Society for Oral Medicine (BSOM) council and had representation from consultant trainers, recent former trainees, and specialty registrars in OM.

Since the assumed medical competencies were derived from undergraduate medical degree courses and codified in the immediate post-qualification years of medical training (Foundation Training), the first stage of the process was to review the collection of current medical competencies described by the UK Foundation Program Office (UKFPO) in its document "The UK Foundation Programme Curriculum." ${ }^{3}$ The UKFPO facilitates the development and operation of the medical foundation program on behalf of the four UK health departments (England, Wales, Scotland, and Northern Ireland) in the National Health Service (NHS). The foundation program runs over two years; after the first year of hospital practice, a newly qualified, provisionally registered doctor can gain full registration with the GMC. The foundation program curriculum includes a syllabus that describes the medical and other competencies to be gained in medical foundation training, from which 20 medical competencies to be considered by the group were derived. A further 15 competencies were taken from the GMC document "Outcomes for Provisionally Registered Doctors with a Licence to Practice."4 
These 35 competencies were to be considered by the working group, and a recognized valid method was needed to achieve agreement. In 1996, Adler and Ziglio described their use of the Delphi technique, building on the description by Brown at the Rand Corporation in 1968. ${ }^{5,6}$ The Delphi method is a well-established method for generating curriculum content as this process represents expert consensus and can be considered an evidence-based process in educational research. ${ }^{7}$ The technique uses face-toface meeting and voting on each item successively until a consensus is reached. A modification of this method has been described in which mail or email is used to achieve consensus. ${ }^{8}$ The method used by this OM working group was a combination of the two: face-to-face meetings supported by email circulation of documentation and discussion. Following advice from the chair of the previous working group (that developed the 2010 curriculum), the level of consensus was set at $90 \%$ or above to accept or reject a proposed medical competency.

\section{Results}

Proceeding through the timeline (Table 1), the working group considered all 35 medical competencies derived from medical training curricula. For each of the proposed medical competencies, the choices were to accept, reject, or note that the competency was already included in the GDC OM specialty training curriculum.

A summary of the outcomes is shown in Table 2, Table 3, and Table 4. Table 3 includes the rationale

Table 1. Timeline of modified Delphi approval/rejection of proposed medical competencies for oral medicine

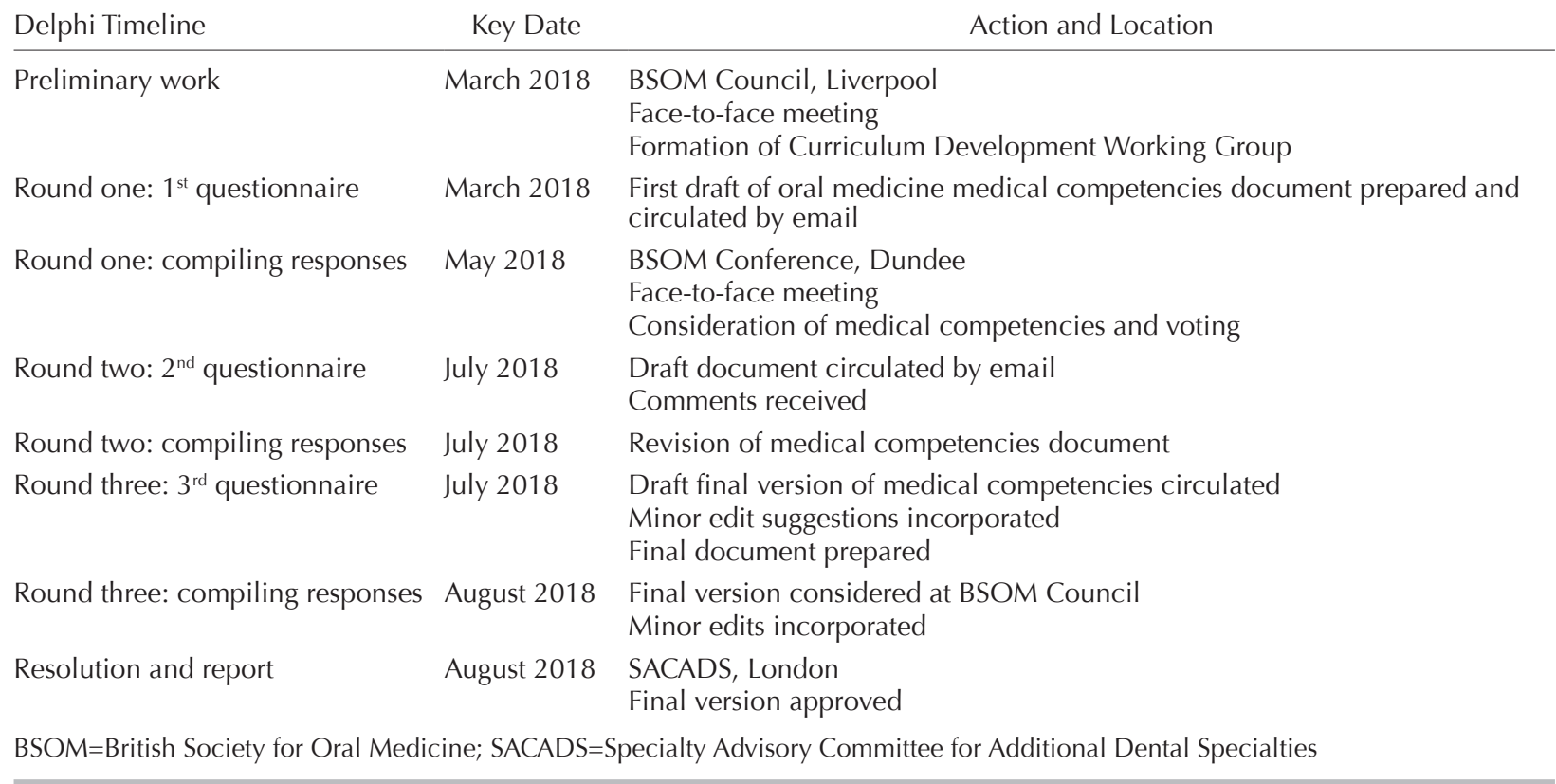

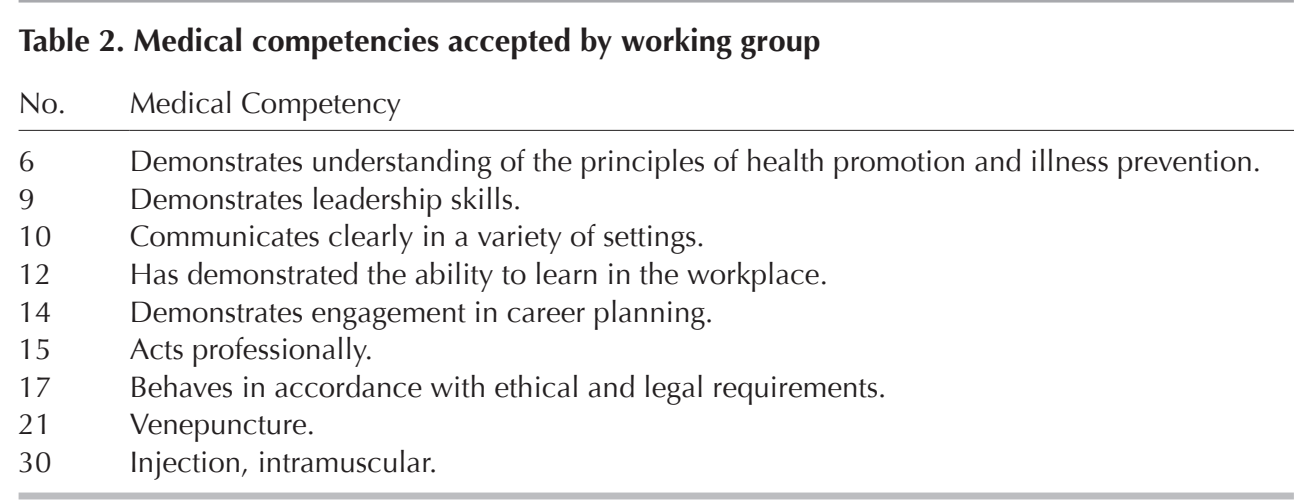




\section{Table 3. Medical competencies rejected by working group}

\begin{tabular}{|c|c|c|}
\hline No. & Medical Competency & Reason for rejection \\
\hline 7 & Manages palliative and end of life care under supervision. & Inpatient management \\
\hline 22 & IV cannulation. & $\begin{array}{l}\text { Inpatient management or for dental sedation } \\
\text { (in oral surgery or special care dentistry) }\end{array}$ \\
\hline 23 & Prepare and administer IV medications and injections. & Inpatient management \\
\hline 24 & Arterial puncture in an adult. & \\
\hline 25 & Blood culture from peripheral sites. & \\
\hline 26 & Intravenous infusion including the prescription of fluids. & \\
\hline 27 & Intravenous infusion of blood and blood products. & \\
\hline 28 & Injection of local anaesthetic to skin. & In oral surgery or oral \& maxillofacial surgery \\
\hline 29 & Injection, subcutaneous (e.g., insulin or LMW heparin). & Inpatient management \\
\hline 31 & Perform and interpret an ECG. & \\
\hline 32 & Perform and interpret peak flow. & \\
\hline 33 & Urethral catheterization (male). & \\
\hline 34 & Urethral catheterization (female). & \\
\hline
\end{tabular}

\section{Table 4. Competencies considered already to be present in oral medicine (OM) curriculum or overall General Dental}

Council (GDC) guidance

No. Medical Competency Where Present

1 Recognizes, assesses, and initiates management of the OM curriculum: B6, Medical emergencies acutely ill patient.

2 Recognizes, assesses, and manages patients with longterm conditions.

3 Obtains history, performs clinical examination, formulates differential diagnosis and management plan.

$4 \quad$ Requests relevant investigations and acts upon results.

5 Is trained and initiates management of cardiac and respiratory arrest.

8 Works effectively as a team member.

11 Recognizes and works within limits of personal competence.

13 Keeps practice up-to-date through learning and teaching. 16 Delivers patient-centered care and maintains trust.

18 Makes patient safety a priority in clinical practice.

19 Contributes to quality improvement.

20 Performs procedures safely.

35 Airway care including simple adjuncts (e.g., Guedel airway or laryngeal masks).
OM curriculum: B4, Interface of oral \& systemic disease

OM curriculum: A1, History taking

OM curriculum: A2, Clinical examination

OM curriculum: A3, Investigations

OM curriculum: A4, Patient management

OM curriculum: A4, Patient management

OM curriculum: B6, Medical emergencies

OM curriculum: generic components-teamworking GDC UK: standards for the dental team

OM curriculum: generic components-teaching and training GDC UK: standards for the dental team

OM curriculum: generic components-patient safety OM curriculum: generic components-quality improvement OM curriculum: generic components-patient safety OM curriculum: B6, Medical emergencies

Sources: OM curriculum appears in Felix DH, Atkin PA, Nolan A, et al. Specialty training curriculum for oral medicine. General Dental Council, UK. 2010. At: www.gdc-uk.org/api/files/OralMedicineCurriculum.pdf. Accessed 1 May 2019. Source for GDC UK is General Dental Council. GDC standards for the dental team. 2013. At: standards.gdc-uk.org/Assets/pdf/Standards $\% 20$ for $\% 20$ the $\% 20$ Dental\% 20 Team.pdf. Accessed 1 May 2019.

for why a medical competency was rejected (typically related to inpatient management outside of OM practice or being relevant to a different dental specialty). Table 4 shows where in the OM curriculum a medical competency was already present or formed part of all GDC dental registrants' practice. Of the 35 competencies considered, nine were accepted, 13 were rejected, and 13 were considered to already be represented in the OM curriculum.

\section{Discussion and Conclusion}

The medical competency working group agreed that the use of a modified Delphi method was 
effective in helping to define those medical competencies to be adopted by the SACADS for current OM training and recommended for inclusion in a future update of the UK GDC curriculum for OM specialty training. In the short term, the BSOM subgroup has agreed to circulate the competencies to the training program directors looking after the specialty registrars currently in training in the UK and Ireland. This list of medical competencies may also be of use for other OM training programs outside of the UK and Ireland. The modified Delphi method described and successfully used for the OM curriculum medical competencies may be readily adopted by other dental specialty curriculum working groups addressing similar problems.

\section{Acknowledgments}

We thank all the participants of the OM curriculum medical competencies working group: Prof. John Gibson, Aberdeen; Dr. Amanda Willis, Belfast; Dr. Jon Higham, Birmingham; Dr. Andrea Richards, Birmingham; Dr. Konrad Staines, Bristol; Dr. Clare Healy, Dublin; Dr. Alan Mighell, Leeds; Dr. Bijay Rajlawat, Liverpool; Dr. John Buchanan, Queen Mary, London; Dr. Emma Hayes, University College, London; Dr. Meena Rudralingam, Manchester; and Dr. Ann Hegarty, Sheffield.

\section{Disclosure}

The authors reported no conflicts of interest.

\section{REFERENCES}

1. Felix DH, Atkin PA, Nolan A, et al. Specialty training curriculum for oral medicine. General Dental Council. 2010 At: www.gdc-uk.org/api/files/OralMedicineCurriculum. pdf. Accessed 1 May 2019.

2. General Dental Council. Standards for specialty education. 2019. At: www.gdc-uk.org/api/files/Standards for Specialty Education.pdf. Accessed 1 May 2019.

3. National Health Service. The UK foundation programme curriculum. At: www.foundationprogramme.nhs.uk/ sites/default/files/2018-07/Curriculum_0.pdf. Accessed 1 May 2019.

4. General Medical Council. Outcomes for provisionally registered doctors with a licence to practice. At: www.gmc-uk.org/-/media/documents/outcomes-forprovisionally-registered-doctors-jul15_pdf-61407158. pdf. Accessed 1 May 2019.

5. Adler M, Ziglio E. Gazing into the oracle: the Delphi method and its application to social policy and public health. London: Kingsley Publishers, 1996.

6. Brown B. Delphi process: a methodology used for the elicitation of opinions of experts. Rand Corporation. 1968. At: www.rand.org/content/dam/rand/pubs/papers/2006/ P3925.pdf. Accessed 1 May 2019.

7. Mitzman J, King AM, Fastle RK, et al. A modified Delphi study for development of a pediatric curriculum for emergency medicine residents. AEM Educ Train 2017;1(2):140-50.

8. University of Illinois Extension. Modified Delphi technique. At: www.communitydevelopment.uiuc.edu/sp/ Step6/DelphiTechnique.pdf. Accessed 1 May 2019. 